\title{
ORIENTACIÓN DE LA EDUCACIÓN EN EL SIGLO XXI
}

Irma Aragón Andrade

\begin{abstract}
RESUMEN
El presente artículo trata sobre cuáles son las habilidades que debe considerar la educación como importantes para poder cumplir su función en forma adecuada. La educación del Siglo XXI no debe estar dirigida a la transmisión de conocimientos y de informaciones sino a desarrollar la capacidad de producirlos y utilizarlos. Diferentes especialistas y organismos internacionales llegaron a un acuerdo $y$ señalaron que el pensamiento crítico, la creatividad, la comunicación y la cooperación son las habilidades esenciales a ser consideradas en la educación del Siglo XXI.
\end{abstract}

\section{PALABRAS CLAVE}

Pensamiento crítico y la resolución de problemas, comunicación, colaboración, creatividad e innovación.

\section{ABSTRACT}

The present article addresses what skills education should consider as important in order to fulfill adequately its function. Education in the $21^{\text {st }}$ Century should not be aimed at the transmission of knowledge and information but at developing the capacity to produce and use them. Different experts and international bodies reached an agreement and pointed out that critical thinking, creativity, communication and cooperation are the essential skills to be considered in $21^{\text {st }}$ Century education.

\section{KEYWORDS}

Critical thinking and problem solving, communication, collaboration, creativity and innovation.
$\mathrm{N}$ os encontramos en el Siglo XXI llamado la era de la información. ¿Qué implicancias puede tener este siglo en relación a la educación?

El Siglo XXI tiene como característica principal el avance y expansión de la digitalización y el control de la información a nivel global, lo que se está dando por la expansión de nuevos dispositivos de almacenamiento de datos, la masificación de la telefonía móvil, el bajo costo para acceder al servicio de Internet y el establecimiento de las redes sociales que permiten la mensajería instantánea. Los cambios se producen en todas las sociedades y en sus diferentes dimensiones, en unas sociedades más que en otras; estos cambios han hecho que los desafíos educativos actuales sean distintos a los del siglo pasado.

La educación es la adquisición de conocimientos para tener oportunidades profesionales y laborales, el poder desarrollar nuestras capacidades y lo mejor que tiene cada uno de los individuos que conforman la sociedad, en beneficio de sí mismos y de la sociedad en general; por lo tanto, la educación es para obtener resultados en el presente pero más en la preparación que hace de ese individuo y su futuro.

El futuro en un mundo globalizado con acceso inmediato a la información, con una tecnología cada vez más avanzada y especializada en todos 
los ámbitos, lo que va a producir (y está produciendo) cambios muy rápidos en todo nivel. Las exigencias actuales a nivel laboral son diferentes con respecto al siglo XX, siendo la educación una condición necesaria para garantizar la competitividad, empleabilidad y desempeño ciudadano, existen cada vez más dificultades para generar empleos e ingresos para la población. En este sentido, el problema ya no se reduce exclusivamente a la dificultad para transformar la educación desde el punto de vista de sus modelos de organización y gestión, sino a las dificultades que tienen los diferentes modelos de gestión educativa para proyectarse al futuro y todo lo que define la función de transmisión que poseen la educación y la escuela.

La aparición de las nuevas tecnologías ha producido un fenómeno comparable al que produjo la invención de la imprenta. Toda la información que no circule por los circuitos creados por estas tecnologías tendrá una existencia corta, como tuvieron la información y el conocimiento que no fue incorporado al libro o al documento escrito a partir de la expansión de la imprenta. Esta concentración de conocimientos e informaciones en los circuitos de las nuevas tecnologías -como es el caso de Internet, por ejemplo- explica la necesidad de incorporar adecuadamente la dimensión tecnológica en las políticas educativas democráticas. El no hacerlo está excluyendo a todos los que queden fuera del dominio de los códigos que permitan manejar estos instrumentos; además se está dando a nivel mundial la concentración del acceso a las nuevas tecnologías en ciertas regiones y sectores de la población.

Se debe considerar que la educación no debe estar dirigida a la transmisión de conocimientos y de informaciones sino a desarrollar la capacidad de producirlos y utilizarlos. A diferencia de la educación del siglo XX, en la que los conocimientos adquiridos durante la época escolar y universitaria no se volvían tan rápidamente obsoletos, ahora hay la posibilidad de acceder a gran cantidad de información y de datos que nos obligan a seleccionar, organizar y procesar la información para poder utilizarla; por lo tanto, ha habido cambio de objetivos en la educación del presente siglo. Las actuales tendencias pedagógicas que ponen énfasis en los aspectos meta curriculares, son los conocimientos sobre los conocimientos: como obtener información, como pensar correctamente, nos permiten comprender y desarrollar eficiente y conscientemente las tareas para aprender cosas nuevas y usar nuestros conocimientos para resolver problemas.

Si el objetivo de la educación consiste en transmitir los conocimientos para lograr los conocimientos -llamados también de orden superior-el papel de los docentes no puede seguir siendo el mismo; su función se resume, desde este punto de vista, en la tarea de enseñar a aprender a aprender, lo cual se contrapone al actual modelo de relación entre profesor y alumno, donde el alumno no aprende las operaciones cognitivas destinadas a producir más conocimiento sino las operaciones que permiten triunfar en el proceso escolar.

El papel del docente se debe dar como un acompañante cognitivo, en donde él debe ser capaz de exteriorizar un proceso mental que generalmente es implícito, mediante el desarrollo de actividades destinadas a hacer explícitos los comportamientos que van a ser posible la comprensión y solución de problemas, mediante la incorporación de las operaciones mentales que permitan esto, es decir mediante procesos metacognitivos se va a aprender a aprender.

\section{LAS CUATRO CS}

La educación del Siglo XXI debe orientarse al desarrollo de las habilidades que los estudiantes necesitan para tener éxito en el trabajo y en su vida en general. Durante el siglo XX las habilidades que se consideraban básicas para el trabajo eran: la lectura, la escritura y la aritmética.

A diferencia de ellas y de acuerdo a las características y a las necesidades sociales que se presentan en la sociedad del Siglo XXI, la educación debe orientarse al desarrollo del pensamiento crítico, comunicación, colaboración y creatividad (4 Cs). Este enfoque se inició en el año 2002, y fue promovido por la Asociación para las habilidades del Siglo XXI-Partnership for 2lst Century Skills (en adelante P21)- que es una 
organización de Estados Unidos que agrupa entre otros a los siguientes miembros: (i) El departamento de educación de EE.UU. (United States Department of Education), (ii) diversas organizaciones (AOL Time Warner Foundation, Apple Computer, Inc., Cable in the Classroom, Cisco Systems, Inc., Dell Computer Corporation, Microsoft Corporation) y (iii) personas individuales. El objetivo de P2l es fomentar un diálogo nacional sobre la importancia de las habilidades del siglo XXI para todos los estudiantes. Actualmente, existen investigaciones que exploran como se pueden integrar las cuatro Cs en el entorno del aprendizaje.

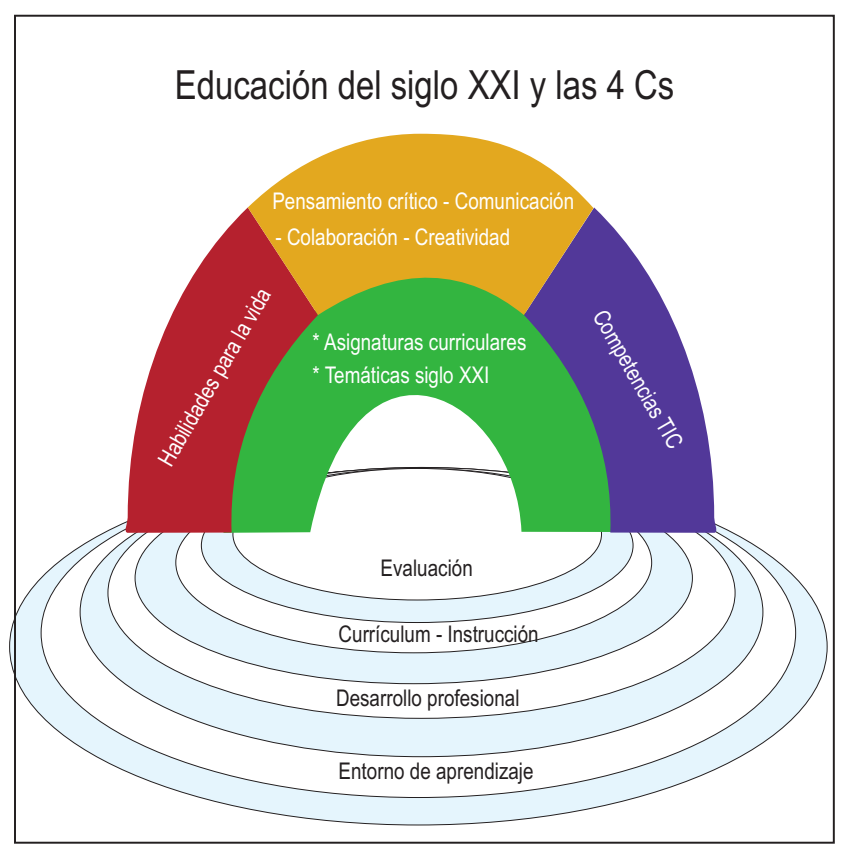

Según lo señalado por Partnership for 2lst Century Skills, en la figura se representa el marco P21, una serie de conocimientos, habilidades y actitudes necesarias para todo estudiante del siglo XXI (a colores) y también aquellos sistemas de apoyo con los que el docente cuenta en su práctica diaria (tal y como se representa en la parte inferior de la figura). Así, la formación del estudiante del siglo XXI, según el marco P21, engloba:

Asignaturas curriculares y temas básicos del siglo XXI. Habilidades para que los alumnos sean independientes y estén preparados para la vida cada vez más compleja. Los alumnos tienen que estar preparados para trabajos que no existen y herramientas que aún no se han desarrollado. Dichas habilidades incluyen:

1.- El pensamiento crítico y la resolución de problemas.

2.- La comunicación.

3.- La colaboración.

4.- La creatividad y la innovación.

Competencias de aprendizaje e innovación. El dominio del inglés, lectura, idiomas, artes, matemáticas, economía, ciencias, geografía, historia, gobierno y civismo, pero también promover la comprensión de temas interdisciplinarios como pueden son: (i) conciencia global, (ii) financiera, económica, empresa y alfabetización empresarial, (iii) alfabetización cívica, (iv) educación para la Salud y (v) alfabetización ambiental.

Competencia en manejo de las Tecnologías de la Información y Comunicación (TIC's). Hoy en día, vivimos en un entorno de tecnología y medios de comunicación marcada por el acceso a abundante información y los rápidos cambios en las herramientas tecnológicas. Así, los ciudadanos del siglo XXI deben ser capaces de adquirir una gama de habilidades tales como la alfabetización de la información, alfabetización mediática y, en definitiva, el dominio de las TIC.

Habilidades para la vida personal y profesional. Los entornos de trabajo del siglo XXI requieren de habilidades profesionales como las que se mencionan a continuación: 1. flexibilidad y adaptabilidad, 2. iniciativa y autonomía, 3 . habilidades sociales y culturales, 4 . productividad y rendición de cuentas, y 5 . liderazgo y responsabilidad.

Las evaluaciones, el currículum e instrucción, el desarrollo profesional y el entorno de aprendizaje son herramientas y recursos que el docente tiene a su disposición para producir los resultados del proceso educativo que se esperan y poder así 
obtener la educación de calidad requerida en este siglo XXI.

Explicaremos en forma muy corta a que se refiere las cuatro Cs:

El pensamiento crítico en la educación, la enseñanza del pensamiento crítico y la resolución de problemas de manera eficaz en el aula es vital para los estudiantes. Aprender el pensamiento crítico lleva a los estudiantes a desarrollar otras habilidades como mayor nivel de concentración, la capacidad de análisis más profundo y la mejora de procesamiento del pensamiento.

El pensamiento crítico y resolución de problemas es definido para la Web P21 como:

La razón efectiva, el utilizar varios tipos de razonamiento (inductivo, deductivo, etc.) según sea apropiado a la situación pensando en el uso de sistemas, analizar cómo las partes de todas interactúan entre sí para producir los resultados globales en sistemas complejos.

El pensamiento crítico es lo más importante en las cuatro Cs, y se vincula íntimamente con la creatividad, porque las dos están íntimamente ligadas; el pensamiento tiene que ser crítico y creativo porque se produce, evalúa, genera y juzga los productos del pensamiento.

La comunicación en la educación, es expresar pensamientos, comunicar instrucciones coherentes, motivar a los otros a través del habla. Estas habilidades siempre han sido valoradas pero ahora son más importantes que en el Siglo XX debido a que ahora los estudiantes deben ser capaces de analizar en forma eficaz una mayor cantidad de información, además de poder comunicarse con claridad y eficacia en variedad deidiomas.

Según P21 define la capacidad de comunicación como la articulación de pensamientos e ideas de forma eficaz, ya sea en forma oral o escrita y habilidades de comunicación no verbal. También el escuchar en forma eficaz para descifrar el significado de lo que nos quieren expresar, en donde se incluye conocimientos, valores, actitudes eintenciones.
Usar la comunicación para una variedad de propósitos (por ejemplo, para informar, instruir, motivar, y persuadir). El uso de múltiples medios y tecnologías y comunicarse eficazmente mediante diferentes idiomas en diversos ambientes culturales.

El comunicarse en forma efectiva está relacionado con el trabajar en diferentes equipos, es colaboración, teniendo el compromiso necesario para lograr una meta común y asumiendo la responsabilidad compartida para el trabajo cooperativo.

La colaboración, es una habilidad que mediante el trabajo colaborativo puede lograr resultados significativos y eficaces. Se ha encontrado que los grupos en circunstancias adecuadas son más inteligentes que las personas más inteligentes que los conforman. La diversidad y características personales de los miembros que conforman un grupo va a aportar al grupo diferentes perspectivas individuales y culturales que enriquecen el trabajo cooperativo; por lo tanto, se generara mayor conocimiento. El término colaboración se define según la Web P2l, este concepto hace referencia a los siguientes aspectos: colaborar con los demás, demostrar capacidad para trabajar de manera eficaz y respetuosa con diversos equipos, mostrar voluntad para comprometerse con los demás en la consecución de un objetivo común, asumir una responsabilidad compartida en el trabajo colaborativo, pero también en el valor de las contribuciones individuales realizadas por cada miembro del equipo.

La creatividad. En el mundo competitivo actual y la automatización de tareas, la capacidad de innovación y el espíritu creativo se están convirtiendo rápidamente en requisitos indispensables para el éxito personal y profesional. El término creatividad en la Web P2l la define de la siguiente forma:

Al pensar de forma creativa, se crean nuevas ideas mediante la utilización de una amplia gama de técnicas de generación de ideas y se elabora, perfecciona, analiza y evalúa ideas originales para mejorar y maximizar los esfuerzos creativos. 
Al trabajar de forma creativa con los demás se desarrolla, implementa y comunica nuevas ideas a los demás de manera efectiva. Se es abierto y receptivo a nuevas y diversas perspectivas; se incorpora los aportes del grupo y la retroalimentación en el trabajo. Se percibe el fracaso como una oportunidad para aprender; se entiende que la creatividad y la innovación se desarrollan a largo plazo: los pequeños éxitos y los frecuentes errores contribuyen a la innovación. La innovación hoy en día tiene un componente social y requiere de la capacidad de adaptación, liderazgo, trabajo en equipo y habilidades interpersonales. Cada vez más, en la actualidad la capacidad de innovar está vinculada a la capacidad para conectar con los demás y con la facilidad para la comunicación y la colaboración.

\section{CONCLUSIÓN}

Por lo tanto las estrategias de aprendizaje deben tener como objetivo el desarrollo de las $4 \mathrm{Cs}$, mediante la aplicación de técnicas que permitan el desarrollo del pensamiento crítico, comunicación, colaboración y creatividad en forma individual o integrada. Los materiales a utilizarse dentro de las diversas técnicas, no se diferencian en el desarrollo de las $4 \mathrm{Cs}$, lo que cambia es el fin que queremos lograr con ellas y las habilidades que queremos desarrollar. El país que tiene la mejor educación es Finlandia porque se basa en el desarrollo del pensamiento crítico y creativo; el logro de esto mediante la colaboración y la utilización de los diferentes medios de comunicación para llevarlo a cabo en forma eficiente y en especial porque los docentes están capacitados para su desarrollo porque cuentan con el apoyo económico -un alto porcentaje del presupuesto nacional se invierte en educaciónpara poder capacitarse, implementar diferentes estrategias de aprendizaje en sus alumnos; además, consideran la educación como un aspecto fundamental para el crecimiento y desarrollo de la economía de su país. En el Perú podemos establecer comparación con la educación de Finlandia y la causa principal sería que la inversión en educación ha descendido durante la última década, por lo cual no se le está considerando como un factor de desarrollo importante.

\section{REFERENCIAS}

Jopen Sánchez, Guillermo; Gómez Velarde, Walter. Sistema educativo peruano: Balance y agenda pendiente. Documento de trabajo № 379 (setiembre 2011), PUCP. La educación del siglo XXI. Una apuesta de futuro. Fundación de la innovación. Bankinter Accentur, 2011.

Montoya Maya, Javier. Estrategias didácticas para fomentar el pensamiento crítico en el aula. Revista virtual. Universidad Católica del Norte № 25.

Tedesco, Juan Carlos (2011). Los desafíos de la educación básica en el siglo XXI. Revista Iberoamericana de Educación, № 55 (2011), pp. 31-47.

www.p21.org/about-us/p21-framework 